\title{
Percutaneous Transluminal Angioplasty in the Treatment of Diabetic Foot
}

\section{Zhengjun Cuil ${ }^{1 *}$, Imran Khan², Gao Ya ${ }^{3}$, Guo Pengfei ${ }^{3}$, Qing-nan Meng ${ }^{3}$, Rongqiang Yang ${ }^{4}$ and Xiaobo Wang ${ }^{5}$}

${ }^{1}$ Professor and HOD, Department of Burn and Repair Reconstruction Surgery, Hospital of Zhengzhou University, China

${ }^{2}$ Doctoral Student, Department of Plastic Surgery, Hospital of Zhengzhou University, China

${ }^{3}$ Attending Doctor, Department of Burn and Repair Reconstruction Surgery, Hospital of Zhengzhou University, China

${ }^{4}$ Resident Doctor, Department of Plastic Surgery, Zhengzhou Central Hospital, Zhengzhou University, China

${ }^{5}$ Resident Doctor, Medical Cosmetology Department, The Third Affiliated Hospital of Zhengzhou University China

*Corresponding author: Zhengjun Cui, Professor and HOD, Department of Burn and Repair Reconstruction Surgery, First affiliated hospital of Zhengzhou University, Zhengzhou, 450000, Henan, China, Email: publication985@gmail.com

\section{Abstract}

Background: Diabetic foot is the foot tissue damage, ulceration, secondary segmental necrosis in diabetic patients. The prevalence of diabetes is predominantly due to long-term high blood sugar, high blood lipids in the state, the systemic arterial prone to atherosclerosis, lower limb arteries are more prone to endometrial damage, and then there is stenosis or occlusion, distal extremity ischemia, Leading to diabetic foot ulcers.

Objective: To assess the value of percutaneous transluminal angioplasty (PTA) in the surgical treatment of diabetic foot. Methods: Retrospective analysis of 83 cases of diabetic foot patients, 95 limbs, 95 wounds were conscripted from September 2011 to September 2014 in our hospital. Rendering to whether the PTA treatment is not accepted PTA treatment of the conventional treatment group 43 cases (wound 51) and PTA treatment of PTA group 40 cases (44). The granulation growth rate of the wound was intended at 3, 6, 9, and 12 days after debridement. The spell of wound preparation was logged before operation. According to the Wagner classification, the survival rate of the skin graft and the healing of the suture in the 2 groups were statistically analyzed. Statistics of two groups of patients were followed up for six months, the monthly review of $\mathrm{ABI}$, and ulcer recurrence. Data were processed with $\chi^{2}$ test and $t$ test.

Results: The good rate of granulation growth of the wound in the conventional treatment group was slower than that of the control group. The good granulation growth rate of the wound was less than $20 \%$ on the 12 th day after treatment. The granulation growth rate of the wound in the PTA group increased visibly from the 9th day, all grow well. On the 9th and 12th day of treatment, the granulation growth rate of the wound in PTA group was ominously advanced than that in the conventional treatment group $(\mathrm{P}<0.01)$. The time of wound preparation was $(24 \pm 10)$ days in the conventional treatment group, which was significantly higher than that in the PTA group $(15 \pm 3$ ) days, $t=5.709, \mathrm{P}<0.01$. The Wagner $2,3,4$ grade wounds were suggestively greater in the PTA group than in the conventional treatment group $(\chi 2=6.741 \sim 24.498, P<0.01)$. ABI was significantly higher in the PTA group than in the conventional treatment group $(t=5.411 \sim 9.583, P<0.01)$. There 


\section{Diabetes \& Obesity International Journal}

was no recurrence of ulcer in the 4 months before the follow-up. There were 1 and 2 cases of ulcer recurrence in the 5 and 6 months follow-up. There was no recurrence in the PTA group after 6 months follow-up.

Conclusion: PTA is effective in the surgical treatment of diabetic foot patients, and has assured clinical application value.

Keywords: Diabetic Foot; Angioplasty; Skin Transplantation; Wound Healing

\section{Introduction}

Diabetic foot is the foot tissue damage, ulceration, secondary segmental necrosis in diabetic patients [1]. The prevalence of diabetes is predominantly due to long-term high blood sugar, high blood lipids in the state, the systemic arterial prone to atherosclerosis, lower limb arteries are more prone to endometrial damage, and then there is stenosis or occlusion, distal extremity ischemia, Leading to diabetic foot ulcers [2]. At present, there are several approaches of handling of diabetic foot, internal medicine by controlling blood sugar, lowering blood lipids, nutritional support and other methods to improve the basic situations of the body [3]; surgery by intensifying the peripheral blood, debridement, elimination of local edema and other methods to lessen wound and systemic infection. Aforementioned studies have focused on the success rate of percutaneous transluminal angioplasty (PTA), the surgical approach, and the rate of revascularization after surgery, with few scrutinizes of patients with traditional surgical treatment of diabetic foot [4]. This study intends to the above two methods of treatment of diabetic foot patients were paralleled, are abridged below.

\section{Objects and Methods}

Retrospective analysis of 83 cases of diabetic foot patients, 95 limbs, 95 wounds were conscripted from
September 2011 to September 2014 in our hospital. Rendering to whether the PTA treatment is not accepted PTA treatment of the conventional treatment group 43 cases (wound 51) and PTA treatment of PTA group 40 cases (44). The granulation growth rate of the wound was intended at $3,6,9$, and 12 days after debridement. The spell of wound preparation was logged before operation. According to the Wagner classification, the survival rate of the skin graft and the healing of the suture in the 2 groups were statistically analyzed. Statistics of two groups of patients were followed up for six months, the monthly review of ABI, and ulcer recurrence. Data were processed with $\chi^{2}$ test and $t$ test.

\section{Selection Criteria}

Inclusion criteria: (1) the existence of classic lower extremity ischemia, foot ulcers. (2) CT angiography results in line with the diagnosis of diabetic vascular lesions. (3) The patient or his family signed the consent to continue surgical treatment of wound preparation. (4) The patient or his family signed a line of surgery to close the wound.

Exclusion criteria: (1) severe wound infection or laboratory tests to support systemic infection is not controlled. (2) Multisystem failure of organ damage in patients with anesthesia risk, such as heart failure, liver and kidney dysfunction. (3) Coagulation system dysfunction there is bleeding tendency. (4) Other systemic diseases may affect the surgical results or wound healing.

\begin{tabular}{|c|c|c|c|c|c|c|c|c|}
\hline \multirow{2}{*}{ Group } & \multirow{2}{*}{$\begin{array}{c}\text { Case } \\
\text { number }\end{array}$} & \multicolumn{2}{|c|}{ Sex(case number) } & \multirow{2}{*}{$\begin{array}{c}\text { Age (vear, } \\
x \pm s \text { ) }\end{array}$} & \multicolumn{3}{|c|}{ Wagner grade (case number) } & \multirow{2}{*}{$\begin{array}{l}\text { Ankle-brachial } \\
\text { index }\end{array}$} \\
\hline & & Man & Woman & & 1 & 2 & 3 & \\
\hline $\begin{array}{l}\text { Conventional Treatment } \\
\text { group }\end{array}$ & 43 & 28 & 15 & $64 \pm 10$ & 9 & 22 & 20 & $0.31 \pm 0.20$ \\
\hline PTA group & 40 & 23 & 17 & $65 \pm 10$ & 7 & 23 & 14 & $0.32 \pm 0.17$ \\
\hline$\chi^{2}$ value & & - & 0.507 & & - & 0.211 & & - \\
\hline $\mathrm{T}$ value & & - & - & 0.796 & - & - & & 0.258 \\
\hline$P$ value & & 0.476 & - & 0.427 & - & 0.646 & & 0.78 \\
\hline
\end{tabular}

Table 1: Two groups of patients with general information comparison.

Note: PTA is Percutaneous Transluminal Angioplasty.

Clinical data were collected from September 2011 to September 2014 in line with the inclusion criteria of diabetic foot patients in 83 cases, 95 limb, which wound 95. Including
51 males and 32 females, aged $(64 \pm 10)$ years. Ulcers in accordance with Wagner classification in line with 2 to 4 , requiring surgical treatment, there may be healing, including 


\section{Diabetes \& Obesity International Journal}

two $16,345,434$. The ankle-brachial index (ABI) was $0.32 \pm$ 0.19 . According to whether the PTA treatment is not accepted PTA treatment of the conventional treatment group 43 cases (wound 51) and PTA treatment of PTA group 40 cases (44). There was no significant difference between the two groups $(\mathrm{P}>0.05)$.

\section{Treatment}

Patients admitted to hospital after routine control of blood glucose, vasodilator, nutritional peripheral nerves and other basic medical treatment, and wound debridement dressing, removal of necrotic tissue. After debridement of wound every two days, according to conventional methods of dressing. PTA group were treated with PTA after exclusion of surgical contraindication, and the $5 \mathrm{~F}$ arterial sheath (American COOK Company) was inserted into the loach guide wire and $5 \mathrm{~F}$ single curved catheter through the sheath of the femoral artery Company), both with the lower limb arterial angiography. After the lesion target vessel was made clear, the V-18 wire was introduced to open the stenosis or occlusion segment to the distal vascular true lumen, and the balloon catheter was introduced to dilate the stenosis segment. For the superficial femoral artery, the knee popliteal artery dissection or long segment occlusion in patients with stent placement, review angiography confirmed that the vessel opened a good exit device. Perioperative and post-discharge regular oral anti-platelet aggregation drugs to prevent reocclusion after treatment of PTA [5]. Granulation tissue to be wound well, the line II granulation wound skin graft; for the foundation of bone destruction, the full removal of necrotic bone tissue, to be retained on both sides of the soft tissue tension-free suture. Routine use of antibiotics to prevent infection after surgery to continue preoperative medication, interval 2 day dressing. According to the wound after about 14 day stitches. Patients discharged after monitoring their own blood sugar, and application of drugs to control blood sugar. Lower extremity warm, anti-pressure, conventional wear soft friction-free shoes, foot skin ointment application of Vaseline to keep moist. Avoid trauma from recurring. Regular assessment of $\mathrm{ABI}$ and wound with or without recurrence.

\section{Observation Indicators}

\begin{tabular}{|c|c|c|c|c|c|}
\hline Group & Number of wound (a) & The third day & The sixth day & The ninth day & The twelfth day \\
\hline Conventional Treatment Group & 51 & $8(15.7)$ & $8(15.7)$ & $13(25.5)$ & $16(31.4)$ \\
\hline PTA group & 44 & $6(13.6)$ & $7(15.9)$ & $36(81.8)$ & $44(100.0)$ \\
\hline$\chi 2$ value & & 0.079 & 0.001 & 30.008 & 47.81 \\
\hline P value & & 0.779 & 0.976 & $<0.001$ & $<0.001$ \\
\hline
\end{tabular}

Table 2: Group compared to the granulation growth rate of the wound in the treatment period [cases (\%)].

Note: PTA is percutaneous transluminal angioplasty.

Wound granulation growth and wound preparation time: Surgery patients of two groups were started after debridement, the calculation of the 3 th, 6 th, 9 th, $12^{\text {th }}$ days of treatment of wound granulation growth rate. Granulation bright red, full, light touch bleeding, bright red blood; or wound granulation dark red, thin, light touch less bleeding, dark red color. The number of days before the wound preparation was counted.

Skin graft survival and suture healing: According to Wagner classification, statistics group 2 patients with skin graft survival and suture healing. Skin graft survival standard: the surgical area less dry or exudate, transplant skin graft survival more than $75 \%$. Each evaluation was performed by a fixed 2 physician.

ABI: Statistics in two groups of patients were followed up for six months, the monthly review of ABI.

Recurrence of ulcers: Patients of two groups were followed up for six months to observe ulcer recurrence.

\section{Statistical Analysis}

SPSS 19.0 statistical software was used to analyze the count data line $\chi 2$ test, measurement data with that line $t$ test. $\mathrm{P}<0.05$ for the difference was statistically significant.

\section{Results}

\section{Wound Granulation Growth and Wound Preparation Time}

The respectable proportion of granulation growth of the wound in the conventional treatment group was slower than that of the control group. The good granulation growth rate of the wound was less than $40 \%$ on the 12 th day after treatment. The granulation growth rate of the wound in the PTA group augmented evidently from the 9th day, Growth is virtuous. On the 9th and 12th day of treatment, the granulation growth rate of the wound in PTA group was significantly higher than that in the conventional treatment group $(\mathrm{P}<0.01)$ Table 2 . The time of wound preparation was $(24 \pm 10)$ days in the conventional treatment group, which was expressively upper than that in the PTA group (15 \pm 3 ) days, $\mathrm{t}=5.709, \mathrm{P}<0.01$. 


\section{The Skin Graft Survival and Suture Healing}

The number of Wagner 2, 3 and 4 wound in the conventional treatment group was 9,13 and 4 respectively, and the number of healing was 2, 2 and 0 . The numbers of Wagner 2, 3 and 4 wound $0,9,16$, the number of healing were $0,2,5$ months. The number of Wagner 2, 3 and 4 wounds in the PTA group was 7, 19 and 7 respectively. The Wagner 2, 3 and 4 wound numbers were 0,4 and 7 , all healed and healed. $(\mathrm{X} 2=6.741 \sim 24.498$, all $\mathrm{P}<0.01)$.

ABI

ABI was significantly higher in the PTA group than in the conventional treatment group $(\mathrm{P}<0.01)$ Table 3.

\begin{tabular}{|c|c|c|c|c|c|c|}
\hline Group & $\begin{array}{c}\text { The first } \\
\text { month }\end{array}$ & $\begin{array}{c}\text { The second } \\
\text { month }\end{array}$ & $\begin{array}{c}\text { The third } \\
\text { month }\end{array}$ & $\begin{array}{c}\text { The fourth } \\
\text { month }\end{array}$ & $\begin{array}{c}\text { The fifth } \\
\text { month }\end{array}$ & $\begin{array}{c}\text { The sixth } \\
\text { month }\end{array}$ \\
\hline $\begin{array}{c}\text { Conventional Treatment } \\
\text { Group }\end{array}$ & $0.31 \pm 0.24$ & $0.31 \pm 0.22$ & $0.33 \pm 0.26$ & $0.32 \pm 0.22$ & $0.31 \pm 0.24$ & $0.32 \pm 0.20$ \\
\hline PTA group & $0.77 \pm 0.23$ & $0.71 \pm 0.25$ & $0.75 \pm 0.21$ & $0.69 \pm 0.31$ & $0.68 \pm 0.29$ & $0.66 \pm 0.30$ \\
\hline t-value & 9.583 & 8.333 & 8.607 & 5.411 & 6.827 & 6.602 \\
\hline P-value & $<0.001$ & $<0.001$ & $<0.001$ & $<0.001$ & $<0.001$ & $<0.001$ \\
\hline
\end{tabular}

Table 3: Two groups of patients were followed up for six months after discharge in each month ABI $(x \pm s)$.

Note: PTA is percutaneous transluminal angioplasty.

\section{Ulcer Recurrence}

The patients in the conventional treatment group did not relapse 4 months before the follow-up. There were 1, 2 cases of recurrence in the 5 and 6 months follow-up. PTA There was no recurrence in the patients within 6 months.

\section{Typical Cases}

Example 1 male, 57 years old, due to diabetes for 10 years, left foot ulceration more than 2 months into the author unit treatment. Admission diagnosis: type 2 diabetes, diabetic foot Wagner 4, macrovascular disease, peripheral neuropathy.
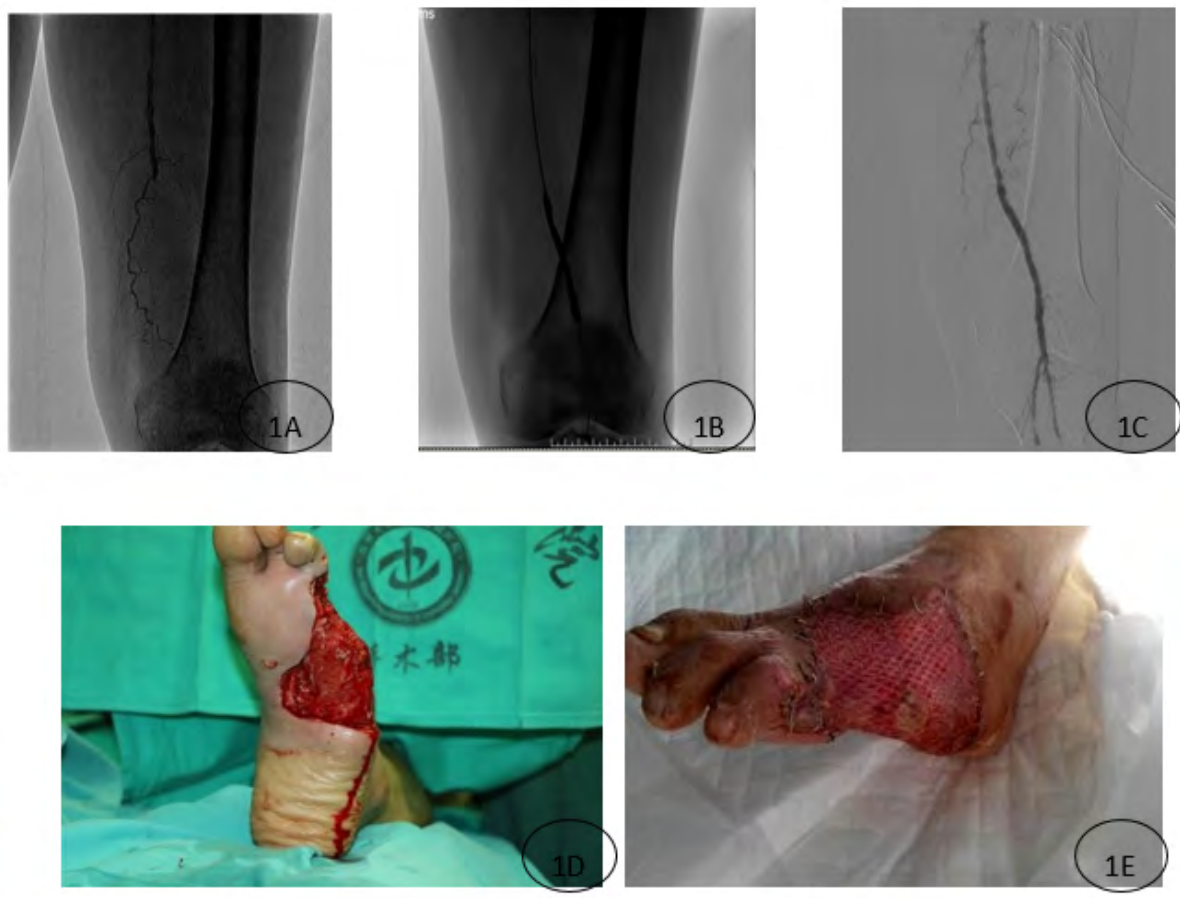

Figure 1: Percutaneous transluminal angioplasty. Treatment 1 Left foot ulceration. 1A. PTA intraoperative arterial angiography showed left lower extremity femoral artery occlusion; 1B. Angiography showed occlusion of the line segment balloon dilatation; 1C. After re-angiography showed superficial femoral artery patency; 1D. Debridement 12d, Full, light touch bleeding, red color; 1E. II period after skin grafting $7 \mathrm{~d}$, skin survival is good. 
After the admission check ABI 0.30, given to control blood sugar, vasodilator, nutritional peripheral nerves and other medical treatment, excluding surgical contraindications after PTA treatment, the sheath into the $5 \mathrm{~F}$ hunter head catheter, arterial angiography showed left lower extremity femoral artery occlusion, $13 \mathrm{~cm}$ after the introduction of guide wire to open the superficial femoral artery occlusion segment, the use of balloon expansion into the stent, again angiography showed superficial femoral artery patency (Figures 1A, 1B, 1C). On the fourth day after PTA, debridement was given, and routine dressing was performed every two days after debridement. 12 days after treatment, there was a new red granulation wound, full, light touch bleeding, and redness. II stage granulation wound skin grafting, conventional dressing after surgery, skin survival is good. See picture 1. Self-monitoring of blood glucose after discharge, wear comfortable friction-free shoes, keep warm, regular followup, after treatment, the monthly follow-up ABI value of $0.72,0.73,0.75,0.73,0.72,0.70$, six months without recurrence Figure 1.
Example 2 male, 64 years old, for more than 10 years of diabetes, left foot toe repeatedly to 3 months into the author unit treatment. Admission diagnosis: type 2 diabetes, diabetic foot Wagner grade 3, macrovascular disease, peripheral neuropathy. After the admission check ABI 0.45 , given control of blood sugar, vasodilator, nutritional peripheral nerves and other medical treatment, excluding surgical contraindications after PTA treatment, the sheath into the $5 \mathrm{~F}$ hunter head catheter, arterial angiography shows the left lower extremity anterior tibial artery stenosis, And then introduced into the stenosis of the guide wire, and balloon dilatation, again arterial angiography showed anterior tibial artery patency. PTA 5 days after debridement, debridement routine dressing every two days. 8 days after treatment wound granulation bright red, full, light touch bleeding, redness. Stage II residual wound closure surgery, conventional dressing after surgery, suture healing, no leakage and so on Figure 2. Self-monitoring of blood glucose after discharge, wear comfortable frictionfree shoes, keep warm, regular follow-up, monthly follow-up after treatment $\mathrm{ABI}$ value of $0.80,0.80,0.82,0.81,0.81,0.80$, six months without recurrence.
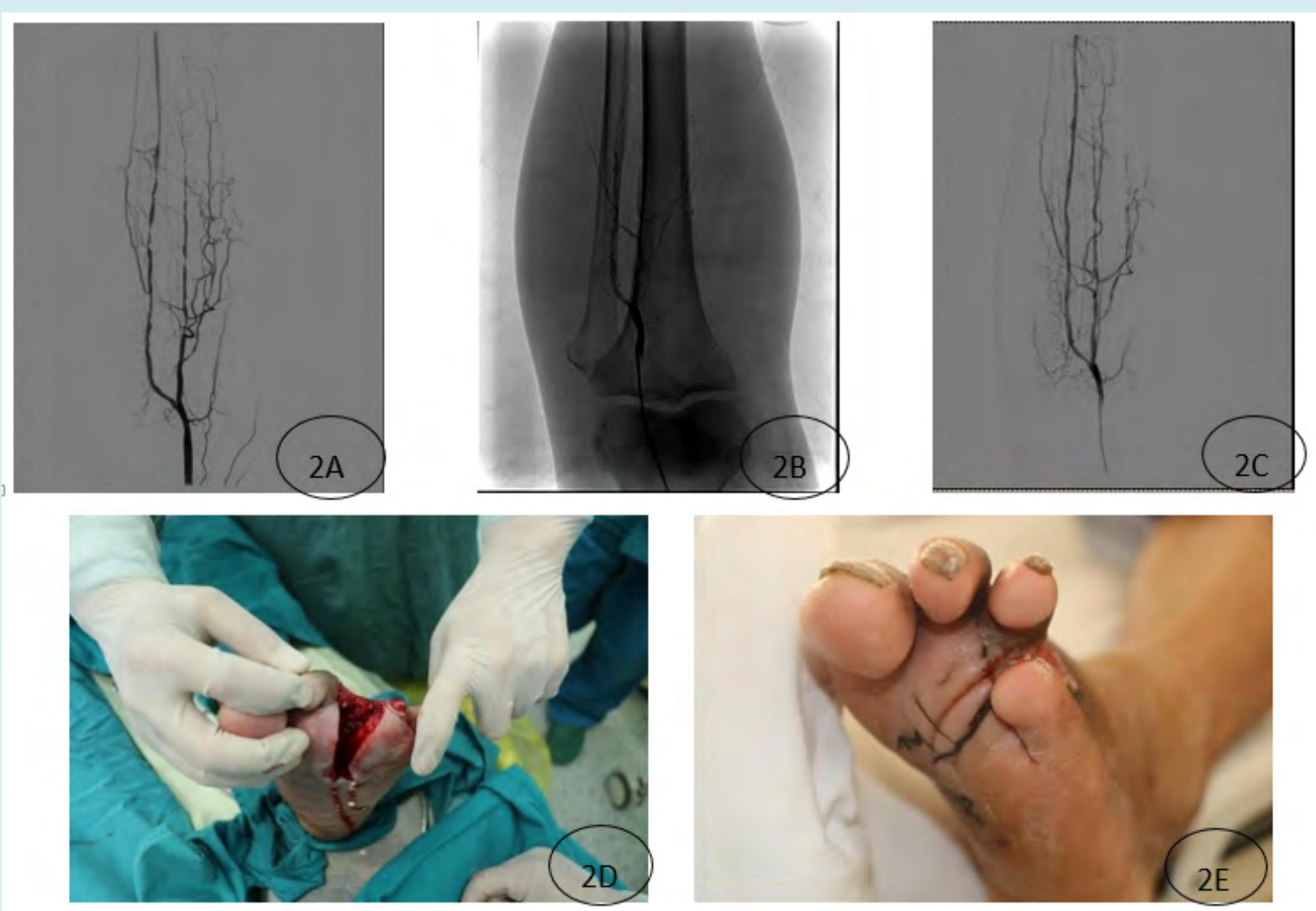

Figure 2: Patients with percutaneous transluminal angioplasty in patients with left foot fourth toe ulceration. 2B. Angiography showed balloon dilatation of the stenosis segment; 2C. Anterior tibial artery patency again; 2D. 8 days after debridement, the granulation was bright red, and the anterior segment of the anterior tibial artery was smooth. , Full, light bleeding, red color; 2E. II residual suture after 14 days, suture healing. 


\section{Diabetes \& Obesity International Journal}

\section{Discussion}

Diabetic vascular lesions mainly violated the small blood vessels below the knee, resulting in insufficient blood supply to the foot. Surgical treatment of diabetic foot ulcers is intended to close the wound, restore patient motor function. Wound closure, foot ulcer infection is the elimination of systemic basis to improve the conditions that guarantee [6]. Diabetic foot patients with ulcers due to foot ulcers, forced to reduce activity, or even long-term bed rest, body nutrition and motor function were decreased [7]. Control foot ulcers and infection, the prevention of sepsis, to promote granulation growth, can be successfully created conditions for II skin graft. In the present study, granulation growth of the wound was significantly better in the PTA group than in the conventional treatment group at the 9th day of treatment, and the time of wound preparation was significantly shorter in the PTA group than in the conventional treatment group, indicating that the PTA group Week began to increase, the wound healing from the role. Diabetic foot wound closure of the main means of free skin grafts, removal of necrotic soft tissue and osteotomy suture, regardless of which method, rich blood supply; adequate nutrients are essential basic conditions [8]. In this study, the wound of the patients belong to chronic refractory wounds, the natural healing is problematic, eliminate the necrotic tissue wound granulation, line II skin graft, to maximize the salvation of limb, long-term recovery motor function. The proportion of Wagner 2, 3 and 4 wounds with wound healing and suture healing was suggestively higher in PTA group than in Wagner group and no reoperation were required. The results showed that the perfusion of lower extremity was significantly amplified after PTA treatment and granulation growth was good. The grafts of the necrotic suture had been healed, which signposted that the stenosis was smooth and the peripheral perfusion increased. The hypoxia of the lower limbs was amended, and the purpose of keeping limbs was attained successfully. Diabetic foot patients after discharge most of the home control their blood sugar, the upshot is often not guaranteed, diabetic foot ulcers occur again, and to prevent the recurrence of ulcers is diabetic foot an important aspect of treatment [9]. In this study, patients with PTA were followed up for a period of time ABI is superior to conventional treatment group; and PTA group patients were followed up for six months without recurrence of ulcers, conventional treatment group were followed up 5,6 months ulcer recurrence cases, indicating PTA treatment Compared with traditional surgical treatment, lower extremity arterial blood supply has improved [10]. As the patient itself with lower extremity vascular disease, traditional surgical treatment of diabetic foot repeated debridement, dressing, etc., the success rate is low, surgical trauma, often lead to amputation. PTA is a minimally invasive surgery, only local anesthesia, with a small risk, quick recovery, repeatability and other advantages, easy to be accepted by patients, has been widely used; and PTA can quickly improve the foot after the blood circulation promote wound healing, reduce the number of operations, shorten the hospital stay, improve the quality of life [11]. According to the follow-up of patients in this study can be considered that the interim effect of interventional therapy is better, but because most of the diabetic patients with high blood sugar, high cholesterol and other related factors exist for a long time, so how to make PTA patients long-term patency, Recurrence is still an urgent problem to be solved [12].

\section{Conclusion}

PTA is effective in the surgical treatment of diabetic foot patients, and has assured clinical application value. PTA is a minimally invasive surgery, only local anesthesia, with a small risk, quick recovery, repeatability and other advantages, easy to be accepted by patients, has been widely used; and PTA can quickly improve the foot after the blood circulation, promote wound healing, reduce the number of operations, shorten the hospital stay, improve the quality of life.

\section{References}

1. Ikem R, Ikem I, Adebayo O, David S (2010) An assessment of peripheral vascular disease in patients with diabetic foot ulcer. Foot (Edinb) 20(4): 114-117.

2. Zhou CG, Dong Weiwei, Zhao Xiaojing (2015) Interventional therapy oflower extremityarteriosclerosis obliterans complicated with diabetic foot and its research progress. Medical Research Quarterly 21(1): 109-111.

3. YE Qiu Ye, SUN Yi Qun (2009) Progress in the treatment of diabetic foot ulcer. Chinese Journal of Injury and Rehabilitation 4(4): 460-466.

4. Longhai Deng, Yin Shiwu (2011) Diabetic foot interventional therapy status and progress. Chinese General Practice 9(10): 1612-1614.

5. Wu Shibai, Yuan Qun, Shi Guzhen (2008) Treatment of diabetic lower limb arterial occlusive sclerosis and foot gangrene by ultrasound thrombus ablation and small balloon intervention. Journal of Second Military Medical University 29(10): 1208- 1212.

6. Elgzyri T, Larsson J, Nyberg P (2014) Early revascularization after admittance to a diabetic foot center affects the healing probability of ischemic foot ulcer in patients with diabetes. Eur J Vasc Endovasc Surg 48(4): 440-446.

7. Xu Zhangrong (2014) Diagnosis and treatment of diabetic foot disease and prevention. Chinese Journal of 
Diabetes \& Obesity International Journal

injury and repair: electronic 9(2): 118-122.

8. Wang Weidong, Liu Lunqin (2010) Clinical efficacy of alprostadil combined with interventional therapy on diabetic foot. Chinese Journal of General Surgery 13(23): 2548-2551.

9. $\mathrm{Xu}$ Zhangrong, Li Xiang (2013) Introduction to the Guidebook on the Management and Prevention of Diabetic Foot in the International Diabetes Foot Work Group 2011. Chinese Journal of Medical Frontiers: Electronic Edition 5(1): 70-72.

10. Wang Jue, Wu Zheng-yang, Zhu Yue-qi (2011) Retrospective analysis of the treatment of severe lower extremity ischemia with long balloon angioplasty in diabetes mellitus. Journal of Interventional Radiology 20(3): 191-195.

11. Craziani L, Silvestro A, Bertone V, Manara E, Andreini R, et al. (2007) Vascular involvement in diabetic subjects with ischemic foot ulcer: a new morphologic categorization of disease severity. Eer J Vase Endovase Surg 33(4): 453460.

12. Xiao Liang, Shen Jing, Huang De-sheng (2011) Analysis of interventional treatment of diabetic lower extremity arterial obstructive disease. Journal of Interventional Radiology 20(3): 218-223. 